This item was submitted to Loughborough's Research Repository by the author.

Items in Figshare are protected by copyright, with all rights reserved, unless otherwise indicated.

\title{
Classical localization and percolation in random environments on trees
}

PLEASE CITE THE PUBLISHED VERSION

PUBLISHER

(C) American Physical Society

LICENCE

CC BY-NC-ND 4.0

REPOSITORY RECORD

Bressloff, P.C., Vincent M. Dwyer, and Michael J. Kearney. 2019. "Classical Localization and Percolation in Random Environments on Trees”. figshare. https://hdl.handle.net/2134/1720. 


\title{
Classical localization and percolation in random environments on trees
}

\author{
Paul C. Bressloff, ${ }^{1}$ Vincent M. Dwyer, ${ }^{2}$ and Michael J. Kearney ${ }^{2}$ \\ ${ }^{I}$ Department of Mathematical Sciences, Loughborough University, Loughborough, Leicestershire LE11 3TU, United Kingdom \\ ${ }^{2}$ Department of Electronic and Electrical Engineering, Loughborough University, \\ Loughborough, Leicestershire LE11 3TU, United Kingdom
}

(Received 30 December 1996)

\begin{abstract}
We consider a simple model of transport on a regular tree, whereby species evolve according to the drift-diffusion equation, and the drift velocity on each branch of the tree is a quenched random variable. The inverse of the steady-state amplitude at the origin is expressed in terms of a random geometric series whose convergence or otherwise determines whether the system is localized or delocalized. In a recent paper [P. C. Bressloff et al., Phys. Rev. Lett. 77, 5075 (1996)], exact criteria were presented that enable one to determine the critical phase boundary for the transition, valid for any distribution of the drift velocities. In this paper we present a detailed derivation of these criteria, consider a number of examples of interest, and establish a connection with conventional percolation theory. The latter suggests a wider application of the results to other models of statistical processes occurring on treelike structures. Generalizations to the case where the underlying tree is irregular in nature are also considered. [S1063-651X(97)12306-6]
\end{abstract}

PACS number(s): 64.60.Cn, 05.40.+j, 05.60.+w

\section{INTRODUCTION}

Statistical problems defined on treelike structures are of interest for two reasons. First, there are a number of physical processes for which the underlying topology is quite naturally treelike in nature. Typical examples are diffusionlimited aggregation, electrodeposition, dielectric breakdown, colloidal aggregation, viscous fingering, and invasion percolation (see, e.g., Refs. [1,2]). Such processes can be modeled in terms of transport occurring in a quenched random environment, leading to anomalous behavior, fractal scaling, and critical phenomena. The second reason why treelike topologies are of interest is that they simplify the analysis compared to a study of the same process defined on a regular lattice. This permits investigations of generic features of interest that can also, in certain cases, be directly relevant to the regular lattice problem in some appropriate limit. For example, it is well known that Cayley trees and Bethe lattices provide insight into the behavior of various processes on both infinite-dimensional lattices and finite-dimensional lattices in the mean-field limit $[3,4]$.

In this paper we consider, in detail, the continuum model of transport on a regular tree defined in Ref. [5]. In this model, the evolution of some species of interest is governed by the drift-diffusion equation, where the drift velocity on each branch of the tree is chosen at random from some specified velocity probability density $\rho(v)$. In other words, the transport takes place in a quenched random environment. An initially localized concentration of species around some selected origin will tend to diffuse away from that origin, but can be hindered in that process by the effects of the random velocity field. If, in the steady state, the concentration remaining at the origin has not decayed to zero, we say the system is localized. If, on the other hand, the concentration at the origin does decay to zero, then we say the system is delocalized. By studying the steady-state solution we have been able to derive exact criteria governing whether, for an arbitrary choice of $\rho(v)$, the system is localized or delocal- ized. The proof is similar to that used to establish theorems regarding the recurrent or transient nature of random walks on treelike structures. However, we have been able to go further, and have also derived integral equations for various distributions of interest.

For particular families of velocity distributions $\rho$ (e.g., Bernoulli, Gaussian, $\Gamma$, etc.) characterized by some parameter(s), it is possible for the system to be either localized or delocalized, depending on the values of the parameter(s). In other words, the system can undergo a phase transition as some parameter is systematically varied, a transition that turns out to be generically first order in nature rather than second order. We present a number of examples of this intrinsically interesting phenomenon. The fact that the criteria we obtain are exact and quite general makes them of wider applicability than simply to the physical model used in their derivation. For example, we establish a close link with various percolation models, showing how the second-order nature of the geometric percolation transition fits in with the first-order behavior of more generalized (two-component) percolation models. We also briefly discuss how certain results can be extended to the case of tree structures that are irregular in nature; e.g., as defined by a genealogical GaltonWatson process with a mean branching number greater than unity.

\section{DRIFT DIFFUSION ON A REGULAR TREE}

Consider an unbounded regular tree $\Gamma$ radiating from a unique origin with branching number $z$ and segment length $L$ (Fig. 1). It is convenient to partition the branches $i \in \Gamma$ of the tree into successive generations. The first generation $S_{1}$ consists of the $z$ branches connected to the origin, the second generation $S_{2}$ consists of the $z^{2}$ subsequent branches connected to the first generation, and so on. The $n$th generation contains $z^{n}$ branches. The set of branches in one generation connected to a segment $i \in \Gamma$ in the preceding generation is denoted by $\mathcal{I}_{i}$. There is a one-to-one correspondence be- 


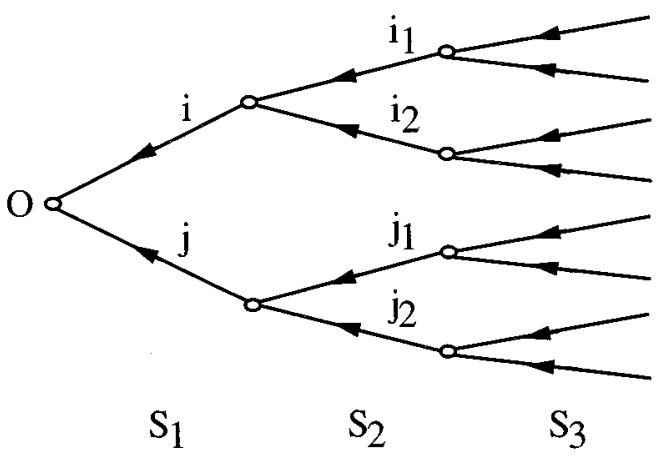

FIG. 1. Topologically biased regular tree with branching number $z=2$ indicating successive generations $S_{1}=\{i, j\}, \quad S_{2}$ $=\left\{i_{1}, i_{2}, j_{1}, j_{2}\right\}$, etc. Also $\mathcal{I}_{i}=\left\{i_{1}, i_{2}\right\}$, etc. Arrows indicate direction of the drift velocity $v_{i}$ on each branch $i$ relative to the origin $O$.

tween vertices of the tree $\Gamma$ (excluding the origin) and branches. Therefore, we shall also use $i$ to denote the vertex whose preceding branch is $i$. We write $i \leqslant j$ if the vertex $i$ is on the shortest (hence every) path from the origin to vertex $j$, and $i<j$ if $i \leqslant j$ and $i \neq j$. Let $|i|$ be the number of branches on the shortest path from the origin to vertex $i$. In particular, for $i \in S_{n},|i|=n$.

We now describe the drift-diffusion equation on the tree $\Gamma$. The concentration $c_{i}(x, t)$ at position $x$ and time $t$ on the $i$ th segment of the tree evolves according to the equation

$$
\frac{\partial c_{i}}{\partial t}=D \frac{\partial^{2} c_{i}}{\partial x^{2}}+v_{i} \frac{\partial c_{i}}{\partial x}, \quad t>0, \quad 0<x<L,
$$

with the end closer to the origin of the tree chosen to be at $x=0$. Here the diffusion constant $D$ is taken to be the same on every branch, and $v_{i}$ is the drift velocity, which is taken to be positive if directed toward the origin, that is, in the negative- $x$ direction. Equation (2.1) is supplemented by boundary conditions expressing continuity of the concentration at a node

$$
\begin{gathered}
c_{i}(0, t)=c_{j}(0, t), \quad i, j \in S_{1}, \\
c_{i}(L, t)=c_{j}(0, t), \quad j \in \mathcal{I}_{i}, \quad i \in \Gamma,
\end{gathered}
$$

and conservation of current through the node

$$
\sum_{k \in S_{1}} J_{k}(0, t)=0, \quad J_{i}(L, t)=\sum_{k \in \mathcal{I}_{i}} J_{k}(0, t),
$$

where

$$
J_{i}(x, t)=-D \partial c_{i} / \partial x-v_{i} c_{i}(x, t) .
$$

In this paper, we are interested in the following classical localization problem: given initial data consisting of a unit impulse located at the origin (or root) of the tree at time $t$ $=0$, what is the asymptotic behaviour of the on-site amplitude $\Phi_{0}(t)$ at the origin? In the absence of drift, it is clear that the on-site amplitude $\Phi_{0}(t)$ decays to zero as $t \rightarrow \infty$ due to the effects of diffusion. In other words, the steady state is delocalized. However, as one switches on a positive inwards drift velocity $v$, one expects the effects of diffusion away from the origin to be counteracted by the drift such that beyond some critical point there is a nonzero steady state, $\lim _{t \rightarrow \infty} \Phi_{0}(t) \neq 0$. The system is then said to be localized. The critical point will depend on the coordination number $z$ since the delocalizing effect of diffusion grows with $z$. An analogous problem was previously investigated within the context of biased random walks on a Bethe lattice, both in discrete time [6] and continuous time [7,8]. A preliminary version of our analysis was presented in Ref. [5]. For convenience, we shall set $D=L=1$ throughout.

In steady state the current vanishes on each segment, $J_{i}$ $\equiv 0$, so that the solution is of the form

$$
c_{i}(x)=A_{i} e^{-v_{i} x} .
$$

The continuity conditions (2.2) imply that the amplitudes $A_{i}$ satisfy the iterative equations

$$
\begin{gathered}
A_{i}=\Phi_{0} \quad \text { for } i \in S_{1}, \\
A_{i} e^{-v_{i}}=A_{j} \quad \text { for all } j \in \mathcal{I}_{i}, \quad i \in \Gamma .
\end{gathered}
$$

Thus the amplitude $A_{i}$ on a given segment $i \in S_{n}, n>1$ may be expressed in terms of the steady-state concentration at the origin $\Phi_{0}$ according to the relation

$$
A_{i}=\left[\prod_{j<i} e^{-v_{j}}\right] \Phi_{0}
$$

Assuming that the initial concentration is normalized to unity, conservation of particle number implies that

$$
\sum_{i \in \Gamma} \int_{0}^{1} c_{i}(x) d x=1
$$

Equation (2.5) then yields the following equation for $\Phi_{0}$ :

$$
\begin{aligned}
\Phi_{0}^{-1}= & \sum_{i \in S_{1}}\left(f\left(v_{i}\right)+g\left(v_{i}\right) \sum_{j \in \mathcal{I}_{i}} f\left(v_{j}\right)\right. \\
& \left.+g\left(v_{i}\right) \sum_{j \in \mathcal{I}_{i}} g\left(v_{j}\right) \sum_{k \in \mathcal{I}_{j}} f\left(v_{k}\right)+\cdots\right),
\end{aligned}
$$

where

$$
f(v)=\frac{\left[1-e^{-v}\right]}{v}, \quad g(v)=e^{-v}
$$

Equation (2.9) expresses $\Phi_{0}^{-1}$ in terms of an infinite series. If this series is convergent then $\Phi_{0}$ has a finite value, and the steady state is localized. On the other hand, if the series diverges, then $\Phi_{0}=0$, and the steady state is delocalized.

The simplest case to analyze is when all drift velocities are the same, $v_{i}=v$ for all $i$. Then Eq. (2.9) reduces to the geometric series

$$
\Phi_{0}^{-1}=f(v) \sum_{p=0}^{\infty} z^{p+1} g(v)^{p} .
$$

Equation (2.11) leads to the following localization criterion: a nonzero steady state occurs, that is, $\lim _{t \rightarrow \infty} \Phi_{0}(t)>0$, if the 
infinite series on the right-hand side of Eq. (2.11) is convergent. This yields the critical velocity

$$
v_{c}=\ln z
$$

and, for $v>v_{c}$,

$$
\lim _{t \rightarrow \infty} \Phi_{0}(t)=\frac{z f(v)}{1-z g(v)}
$$

If $v<v_{c}$ then the asymptotic decay of the delocalized state exhibits conventional behavior, whereas at the critical point $v=v_{c}$ there is anomalous behavior in the form of a critical slowing down [9].

Now suppose that the drift velocities $v_{i}$ are quenched random variables independently and identically distributed from a given probability density $\rho(v)$. Also assume that $v_{i}$ is finite with probability 1 . The right-hand side of Eq. (2.9) becomes a (generalized) random geometric series whose convergence properties determine whether or not the steady state is localized. Naively averaging both sides of Eq. (2.9) with respect to the $v_{i}$ 's, and introducing the notation $\langle X(v)\rangle=\int \rho(v) X(v) d v$ for any measurable function of $v$,

$$
\left\langle\Phi_{0}^{-1}\right\rangle=z\langle f(v)\rangle \sum_{n=0}^{\infty} z^{n}\langle g(v)\rangle^{n}=\frac{z\langle f(v)\rangle}{1-z\langle g(v)\rangle} .
$$

Equation (2.14) shows that $\Phi_{0}^{-1}<\infty$ with probability 1, when $z\langle g(v)\rangle<1$. However, the fact that $\left\langle\Phi_{0}^{-1}\right\rangle \rightarrow \infty$ as $z\langle g(v)\rangle \rightarrow 1$ does not necessarily imply that $\Phi_{0} \rightarrow 0$ (that the steady state becomes delocalized). For the random series, Eq. (2.9) may converge to a random variable whose probability distribution has a long tail with infinite first and higher moments. In Sec. III we shall prove that there exists a sharp first-order phase transition between localized and delocalized states, and determine the location of the transition point for an arbitrary density $\rho(v)$, assuming that each $v_{i}$ is finite with probability 1 . The case of densities $\rho(v)$ for which there is a nonzero probability that $v_{i}$ is infinite, and hence a nonzero probability that there exist broken bonds on the tree (the percolation limit), will be discussed in Sec. IV.

\section{LOCALIZATION-DELOCALIZATION TRANSITION}

\section{A. One-dimensional case $(z=1)$}

When $z=1$, Eq. (2.9) simplifies to the form

$$
\left(\Phi_{0}\right)^{-1} \equiv R=\sum_{n=1}^{\infty} f\left(v_{n}\right) \prod_{m=1}^{n-1} g\left(v_{m}\right)
$$

so that the steady-state concentration is expressed in terms of a random geometric series $R$. Similar series have arisen in a variety of studies of one-dimensional problems in physics $[2,10-13]$ and probability theory [14-16]. The random series $R$ may be generated from the following random difference equation:

$$
Y_{n}^{(N)}=g\left(v_{n}\right) Y_{n+1}^{(N)}+f\left(v_{n}\right), \quad n=1, \ldots, N-1,
$$

with each pair $\left(f\left(v_{n}\right), g\left(v_{n}\right)\right)$ generated independently from $\rho(v)$ and $Y_{N}^{(N)}$ fixed. It can be proven that, if $\langle\ln [g(v)]\rangle<0$ (or $\langle v\rangle>0$ ), then $\lim _{N \rightarrow \infty} Y_{1}^{(N)}=R$ exists with probability 1 and the distribution of $Y_{1}^{(N)}$ converges to that of $R$ independently of $Y_{N}^{(N)}$ [15]. Hence the steady state is localized provided that $\langle v\rangle>0$; that is, the average drift velocity exceeds the critical velocity for localization in the case of uniform one-dimensional drift [see Eq. (2.12)]. On the other hand, if $\langle v\rangle<0$, then $R$ is infinite, and the steady state is delocalized.

In the language of phase transitions, there is a transition from a localized to a delocalized state at the critical points $\langle v\rangle=0$. The critical points determine a phase boundary in the infinite-dimensional space of probability densities $\rho(v)$ that separates the localized and delocalized phases. A characteristic feature of the phase transition is that, as $\langle v\rangle \rightarrow 0^{+}$ in some prescribed fashion, the probability distribution $F$ of $R$ in the localized phase develops a long tail for which all moments are infinite. This is a consequence of the fact that, when the first moment becomes infinite, the system can still be localized. To see this, first note from Eq. (2.14) with $z$ $=1$ that

$$
\int r d F(r)=\frac{\langle f(v)\rangle}{1-\langle g(v)\rangle}
$$

which becomes infinite when $\langle g(v)\rangle=1$. Jensen's inequality $\left\langle e^{-v}\right\rangle \geqslant e^{-\langle v\rangle}$ then implies that $\langle v\rangle \geqslant 0$ when $\langle g(v)\rangle=1$. Assuming the existence of a probability density $\Psi(r)$ such that $d F(r)=\Psi(r) d r$, from Eq. (3.2) we obtain the following Dyson-Schmidt-type integral equation for $\Psi$ :

$$
\Psi(r)=\int_{-\infty}^{\infty} \frac{\rho(v)}{g(v)} \Psi\left(\frac{r-f(v)}{g(v)}\right) d v .
$$

An alternative form of the integral equation (3.4) is obtained by taking Laplace transforms

$$
M(s)=\int_{-\infty}^{\infty} \rho(v) M(g(v) s) e^{-s f(v)} d v,
$$

with

$$
M(s)=\int_{0}^{\infty} e^{-s r} \Psi(r) d r .
$$

It is not generally possible to solve these equations analytically. However, one can determine the asymptotic behavior of $\Psi$ when $r$ is large. For the moment assume that $\rho(v)$ is nonarithmetic, that is, it cannot be written in the form

$$
\rho(v)=\sum_{n=-\infty}^{\infty} p_{n} \delta\left(v-\lambda^{n}\right)
$$

for any $\lambda$ and $\left\{p_{n}\right\}$ such that $\sum_{n=-\infty}^{\infty} p_{n}=1$. Also assume that $\langle v\rangle>0$, and that the first moment of $\Psi$ is infinite so that $\langle g(v)\rangle>1$. It can then be proven [16] that there exist positive constants $a$ and $\sigma$, with $0<\sigma<1$, such that

$$
\Psi(r) \sim a r^{-\sigma-1}
$$

for large $r$, and hence

$$
M(s) \sim 1+b s^{\sigma}
$$


for small $s$. The large- $r$ behavior of $\Psi$ ensures that, if $\sigma$ $>0$, then

$$
F^{*} \equiv \lim _{y \rightarrow \infty} \int_{y}^{\infty} d F(r)=0
$$

That is, the series $R$ of Eq. (3.1) is convergent with probability 1.

Substitution of the asymptotic form for $\Psi$ (or $M$ ) into Eq. (3.4) [or Eq. (3.5)] leads to the equation

$$
\beta(\sigma) \equiv\left\langle g(v)^{\sigma}\right\rangle=1
$$

Useful information concerning the nature of the localizationdelocalization transition can be deduced from Eq. (3.11) $[12,13]$. First note that $\beta(0)=1$ and $\beta(\sigma)$ is a convex function for real $\sigma$. If $\langle v\rangle>0$ then $\beta^{\prime}(0)<0$ and there are two possibilities concerning nontrivial solutions of Eq. (3.11).

(i) $\beta(\sigma)<1$ for any positive real $\sigma$. Since

$$
|\beta(\sigma)| \leqslant \int \rho(v) e^{-\operatorname{Re}(\sigma) v} d v=\beta(\operatorname{Re}(\sigma)),
$$

$\beta(\sigma) \neq 1$ for all $\sigma$; the density $\Psi(r)$ decreases faster than any power of $r$ (finite first moment).

(ii) There exists a single nontrivial real solution $\bar{\sigma}$ of Eq. (3.11). Equation (3.12) implies that $\beta(\sigma) \neq 1$ within the strip $0<\operatorname{Re}(\sigma)<\bar{\sigma}$, but there may exist complex roots of Eq. (3.12) $\sigma=\sigma_{i}$, say, with $\operatorname{Re}\left(\sigma_{i}\right) \geqslant \bar{\sigma}$. For the special class of densities satisfying Eq. (3.7), there exist an infinite number of complex roots with $\operatorname{Re}\left(\sigma_{i}\right)=\bar{\sigma}$ and the asymptotic behavior of $\Psi$ is no longer a simple stable law (see the example of a Bernoulli distribution below). For all other densities $\rho(v)$, all the complex roots satisfy $\operatorname{Re}(\sigma)>\bar{\sigma}$, and the nontrivial real root $\bar{\sigma}$ dominates for large $r$.

Equation (3.11) provides a useful perspective concerning the approach to the transition point. Suppose that $\rho(v)$ depends smoothly on some parameter $\lambda$ such that $\bar{\sigma}(\lambda)>0$ for $\lambda<\lambda_{c}$ and $\lim _{\lambda \rightarrow \lambda_{c}} \bar{\sigma}(\lambda)=0$. In the limit $\lambda \rightarrow \lambda_{c}, \Psi$ ceases to exist (it is no longer normalizable) and the probability $F^{*}$ that $R$ is infinite jumps from $F^{*}=0$ to $F^{*}=1$. Identifying $F^{*}$ as an order parameter, we deduce that the localization-delocalization phase transition is first order. Differentiating the equation $\beta(\bar{\sigma}(\lambda), \lambda)=1$ with respect to $\lambda$ gives

$$
\begin{aligned}
& \int \frac{d \rho_{\lambda}(v)}{d \lambda}[g(v)]^{\bar{\sigma}(\lambda)} d v+\bar{\sigma}^{\prime}(\lambda)\left\langle[g(v)]^{\bar{\sigma}(\lambda)} \ln [g(v)]\right\rangle_{\lambda} \\
& =0
\end{aligned}
$$

Taking the limit $\lambda \rightarrow \lambda_{c}$ in Eq. (3.13) leads to the result $\bar{\sigma}^{\prime}\left(\lambda_{c}\right)\langle v\rangle_{\lambda_{c}}=0$. Since $\lambda_{c}$ is a bifurcation point, it follows that $\bar{\sigma}^{\prime}\left(\lambda_{c}\right)>0$ and hence $\langle v\rangle_{\lambda_{c}}=0$. A simple illustration of these ideas is given in Fig. 2, where $\beta(\sigma)$ is shown for $\rho(v)$ chosen to be a Gaussian with mean $\mu$ and variance $\Delta^{2}$ :

$$
\rho(v)=\frac{1}{\sqrt{2 \pi \Delta^{2}}} \exp \left(-\frac{(v-\mu)^{2}}{2 \Delta^{2}}\right)
$$

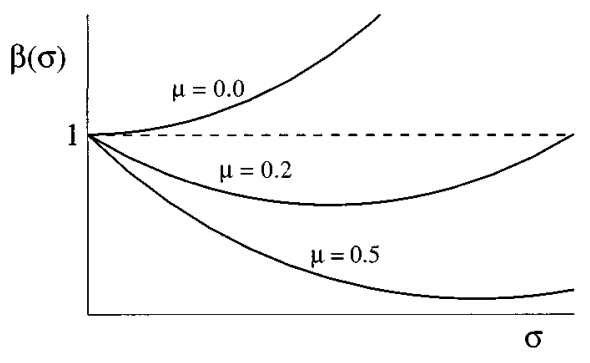

FIG. 2. Plot of the function $\beta(\sigma)$ for a Gaussian distribution with various means $\mu=0,0.2$, and 0.5 , and fixed variance $\Delta^{2}=1$. As $\mu \rightarrow 0$, the nontrivial solution $\bar{\sigma} \rightarrow 0$, where $\beta(\bar{\sigma})=1$, signaling a localization-delocalization phase transition in the case of a onedimensional system $(z=1)$.

Substituting Eq. (3.14) into Eq. (3.11) gives

$$
\beta(\sigma)=\exp \left(-\mu \sigma+\sigma^{2} \Delta^{2} / 2\right) .
$$

For $\mu>0, \beta(\sigma)$ has a single minimum at $\sigma^{*}=\mu / \Delta^{2}$ and $\beta(\bar{\sigma})=1$ for $\bar{\sigma}=2 \mu / \Delta^{2}$. As $\mu \rightarrow 0, \quad \bar{\sigma}(\mu) \rightarrow 0$, and a localization-delocalization phase transition occurs.

As an example of an arithmetic probability density satisfying Eq. (3.7), consider the Bernoulli distribution with density $\rho(v)=p \delta(v-a)+(1-p) \delta(v-\bar{v})$ with $a<0$ and $\bar{v} \rightarrow \infty$. It is clear that the system is localized with probability 1 , since the percolation threshold in one dimension is $p_{c}$ $=1$. Here one can find explicitly the density $\Psi(r)$ satisfying Eq. (3.4) using a similar analysis to Refs. [12,13],

$$
\Psi(r)=(1-p) \sum_{n=0}^{\infty} p^{n} \delta\left(r-r_{n}\right),
$$

where $r_{n}$ satisfies the recursion $r_{n}=g(a) r_{n-1}+f(a)$ with $r_{0}=0$. Hence, $r_{n}=f(a)\left(g(a)^{n}-1\right) /(g(a)-1)$. Since the Bernoulli distribution is arithmetic, the asymptotic behavior of $\Psi$ is no longer a simple stable law. To show this, it is more convenient to look at the asymptotic behavior of the distribution $F(y)=(1-p) \sum_{n=0}^{\infty} p^{n} \theta\left(r_{n}-y\right)$, where $\theta$ is a step function. For large $y, F(y)$ has the asymptotic form $F(y) \sim y^{-\bar{\sigma}} \psi(\xi)$, where $\bar{\sigma}=-\ln p / \ln g(a)$, and $\psi$ is a periodic function of $\xi=[\ln y-\ln f(a)] / \ln g(a)$ with unit period:

$$
\begin{aligned}
\psi(\xi)= & f(a)^{\bar{\sigma}}(1-p) \sum_{n=-\infty}^{\infty} e^{\bar{\sigma}(\xi-n) \ln g(a)} \\
& \times \theta\left(n-\frac{\ln (g(a)-1)}{\ln g(a)}-\xi\right) .
\end{aligned}
$$

One can understand the origins of this periodic behavior by noting that the equation $\beta(\sigma)=1$ reduces to the simple relation $\operatorname{pg}(a)^{\sigma}=1$, which for $a<0$ has the infinite set of complex solutions $\sigma=-\ln p / \ln g(a)+(2 \pi i / \ln g(a)) k$, integer $k$; all solutions have the same real part.

Note that the above results are easily extended to the case of a regular tree with branching number $z>1$ and so-called intergenerational disorder [5]. Here all segments within a generation $n$ have the same drift velocity $v_{n}$ but the sequence $\left\{v_{n}, n \geqslant 1\right\}$ is independently and identically distributed according to a given density $\rho(v)$. The only modification is 
that $g(v)$ is replaced everywhere by $z g(v)$. In particular, Eq. (3.11) becomes $z^{\sigma}\left\langle g(v)^{\sigma}\right\rangle=1$, and the localizationdelocalization transition point now satisfies $\langle v\rangle=\ln z$. The analysis differs considerably from the case $z>1$, in which there is full intragenerational disorder, as we shall now describe.

\section{B. Case $z>1$}

Consider a bounded tree $\Gamma_{N}$ with branching number $z$ $>1$ consisting of $N$ generations, and associate with each segment $i$ a random variable $Y_{i}^{(N)}$ such that (for fixed $Y_{k}^{(N)}$, $\left.k \in S_{N}\right)$

$$
Y_{i}^{(N)}=\sum_{j \in \mathcal{I}_{i}} g\left(v_{i}\right) Y_{j}^{(N)}+f\left(v_{i}\right), \quad i \in \Gamma_{N} .
$$

Equation (2.9) may then be rewritten in the form

$$
\Phi_{0}^{-1}=\sum_{i \in S_{1}} R_{i}, \quad R_{i}=\lim _{N \rightarrow \infty} Y_{i}^{(N)} .
$$

Suppose that $R_{i}$ converges with probability 1 independently of the boundary conditions. The symmetry of the tree then ensures that all variables $R_{i}, i \in S_{1}$, are identically and independently distributed with a probability distribution $F$. The difference equation (3.18) implies that the associated probability density $\Psi$ (assuming it exists) satisfies the Dyson-Schmidt-type integral equation

$$
\begin{aligned}
\Psi(y)= & \int_{0}^{\infty} \prod_{j=1}^{z} \Psi\left(y_{j}\right) d y_{j} \int_{-\infty}^{\infty} \rho(v) \\
& \times \delta\left(y-g(v) \sum_{j=1}^{z} y_{j}-f(v)\right) d v .
\end{aligned}
$$

Laplace transforming Eq. (3.20) gives a corresponding integral equation for the generating function $M(s)$ :

$$
M(s)=\int_{-\infty}^{\infty} \rho(v)[M(s g(v))]^{z} e^{-s f(v)} d v .
$$

Suppose that we expand the generating function $M(s)$ for small $s$ along similar lines to the one-dimensional case such that $M(s) \sim 1+b s^{\sigma}$. Substituting into Eq. (3.21) yields the equation

$$
\beta(\sigma) \equiv\left\langle g(v)^{\sigma}\right\rangle=z^{-1} .
$$

When $z>1, \sigma=0$ is not an allowed root of Eq. (3.22). Therefore, in contrast to the one-dimensional case, the localization-delocalization transition is no longer characterized by the limit $\bar{\sigma} \rightarrow 0$, where $\bar{\sigma}$ is a nontrivial solution of Eq. (3.22). Introduce the index $\sigma^{*} \in[0,1]$ defined according to the property

$$
\beta\left(\sigma^{*}\right)=\min _{0 \leqslant \sigma \leqslant 1} \beta(\sigma) .
$$

Note that $\sigma^{*}$ only depends on the probability density $\rho(v)$. If $z \beta\left(\sigma^{*}\right)>1$, then any solution of $z \beta(\sigma)=1$ must satisfy $\sigma>1$ implying that the first moment of $\Psi$ is finite. On the other hand, if $z \beta\left(\sigma^{*}\right)>1$ then $z\langle g(v)\rangle>1$, and Eq. (2.14) implies that the first moment is infinite. The evident contradiction shows that if $z \beta\left(\sigma^{*}\right)>1$, then the only allowed solution of the integral equation (3.21) for $s>0$ is $M(s)=0$ and the system is delocalized. This gives a heuristic proof of part (ii) of the following theorem.

Theorem 1: Consider the drift-diffusion equation on a regular tree $\Gamma$ with the drift velocities identically and independently distributed on each branch. Assume that the drift velocities are finite with probability one. Let $\beta\left(\sigma^{*}\right)$ be defined according to Eq. (3.23). (i) If $z \beta\left(\sigma^{*}\right)<1$, then the steady state is localized with probability 1 . (ii) If $z \beta\left(\sigma^{*}\right)$ $>1$ then the steady state is delocalized with probability 1 .

Part (i) of this theorem can be established from the integral equation (3.21) in the special case that all drift velocities are restricted to be positive. Since $\beta(\sigma)$ is then a monotonically decreasing function of $\sigma$, it follows that $\sigma^{*}=1$. If $z \beta\left(\sigma^{*}\right)<1$, then all moments of $\Psi$ are finite [cf. Eq. (2.14)], and the system is localized. It follows that for positive drift velocities the system becomes delocalized as soon as the first moment of $\Psi$ becomes infinite, and hence $\Psi$ does not develop a long tail near the transition point.

We shall now present a rigorous proof of theorem 1 that holds for arbitrary distributions $\rho(v)$. Our proof is based on a reformulation of the problem in terms of flows in random electrical or capacitative networks. This then allows us to use some recent results due to Lyons [17] and Lyons and Pemantle [18] concerning random walks in random environments. It is first necessary to introduce some new definitions. For each branch $i \in \Gamma$, set

$$
C_{i}=\left[\prod_{j<i} g\left(v_{j}\right)\right] f\left(v_{i}\right) .
$$

We refer to $C_{i}$ as the "conductance" or "capacity" of branch $i$. Next define a flow $\theta$ on $\Gamma$ to be a set of nonnegative numbers $\left\{\theta_{i}, i \in \Gamma\right\}$, such that, for all $i \in \Gamma$,

$$
\theta_{i}=\sum_{j \in \mathcal{I}_{i}} \theta_{j} .
$$

Define a cutset $\Pi$ to be a finite set of vertices excluding the origin such that every path from the origin to infinity intersects $\Pi$ and such that there is no pair $i, j \in \Pi$ with $i<j$. The shortest distance of a cutset from the origin is written as $|\Pi|=\min \{|i|, i \in \Pi\}$. A special example of a cutset is the $n$th generation $S_{n}, n \geqslant 1$. It follows from Eq. (3.25) that, for any cutset $\Pi$,

$$
\theta(0) \equiv \sum_{i \in S_{1}} \theta_{i}=\sum_{j \in \Pi} \theta_{j} .
$$

If $\theta(0)=1$ then $\theta$ is called a unit flow. Finally, define the energy of a flow for a tree with conductances $C_{i}$ to be

$$
E(\theta)=\sum_{i \in \Gamma} \theta_{i}^{2} C_{i}^{-1}
$$

A useful result concerning flows is the maximum-flow minimum-cut theorem [19]: given a non-negative set $\left\{w_{i}, i\right.$ $\in \Gamma\}$ such that 


$$
\lim _{|\Pi| \rightarrow \infty} \inf \sum_{j \in \Pi} w_{j}>0
$$

then there exists a nonzero flow $\theta$ such that, for all $i \in \Gamma$, $\theta_{i} \leqslant w_{i}$

Proof of theorem 1. Rewrite the expression for the steadystate amplitude at the origin, Eq. (2.9), in the form

$$
\begin{aligned}
\Phi_{0}^{-1} \equiv & C=\sum_{i \in S_{1}} f\left(v_{i}\right)+\sum_{i \in S_{1}} g\left(v_{i}\right) \sum_{j \in \mathcal{I}_{i}} f\left(v_{j}\right) \\
& +\sum_{i \in S_{1}} g\left(v_{i}\right) \sum_{j \in \mathcal{I}_{i}} g\left(v_{j}\right) \sum_{k \in \mathcal{I}_{j}} f\left(v_{k}\right)+\cdots \\
= & \sum_{i \in S_{1}} C_{i}+\sum_{i \in S_{2}} C_{i}+\cdots \\
= & \sum_{i \in \Gamma} C_{i} .
\end{aligned}
$$

Hence, the steady state is localized if and only if the summation over all conductances is finite.

(i) This follows along similar lines to the proof of theorem 1(iii) in Ref. [18]. Define $\bar{\beta}\left(\sigma^{*}\right)=\left\langle f(v)^{\sigma^{*}}\right\rangle$. Suppose $\beta\left(\sigma^{*}\right)<1 / z$. Then

$$
\begin{aligned}
\left\langle\sum_{i \in \Gamma} C_{i}^{\sigma^{*}}\right\rangle & =\sum_{i \in \gamma}\left\langle\prod_{j<i} g\left(v_{j}\right)^{\sigma^{*}}\right\rangle\left\langle f\left(v_{i}\right)^{\sigma^{*}}\right\rangle \\
& =\bar{\beta}\left(\sigma^{*}\right) \sum_{i \in \gamma} \prod_{j<i}\left\langle g\left(v_{j}\right)^{\sigma^{*}}\right\rangle \\
& =\bar{\beta}\left(\sigma^{*}\right) \sum_{n \geqslant 1} \sum_{i \in S_{n}} \beta\left(\sigma^{*}\right)^{n-1} \\
& =\frac{\bar{\beta}\left(\sigma^{*}\right)}{\beta\left(\sigma^{*}\right)} \sum_{n \geqslant 1} z^{n} \beta\left(\sigma^{*}\right)^{n} \\
& =\frac{z \bar{\beta}\left(\sigma^{*}\right)}{1-z \beta\left(\sigma^{*}\right)} \\
& <\infty
\end{aligned}
$$

It follows that $\Sigma_{i \in \Gamma} C_{i}^{\sigma^{*}}<\infty$ with probability 1 , and hence that $C_{i}<1$ for all but finitely many $i \in \Gamma$. Since $C_{i}^{\sigma^{*}} \leqslant C_{i}$ for $C_{i}<1$, we deduce that $\Sigma_{i \in \Gamma} C_{i}<\infty$ and the steady state is localized with probability 1 .

(ii) Suppose $z \beta\left(\sigma^{*}\right)>1$. Proceeding along similar lines to the proof of theorem 1(i) in Ref. [18], one can show that $z \beta\left(\sigma^{*}\right)>1$ implies that, with probability 1 , there exist positive numbers $w_{n}$ such that $\Sigma_{n \geqslant 1} w_{n}<\infty$ and

$$
\lim _{|\pi| \rightarrow \infty} \inf \sum_{j \in \Pi} w_{|j|} C_{j}>0 .
$$

It then follows from the maximum-flow minimum-cut theorem that there exists a flow $\theta$ obeying $\theta_{i} \leqslant w_{n} C_{i}$ for all $i$ $\in S_{n}$ and $n \geqslant 1$. We shall show that the existence of such a flow implies that the total conductance is infinite, and hence that the steady state is localized (cf. the proof of Corollary 4.2 in Ref. [17]). First note that the flow has finite energy. That is,

$$
\begin{aligned}
E(\theta) & =\sum_{j \in \Gamma} \theta_{j}^{2} C_{j}^{-1}=\sum_{n \geqslant 1} \sum_{j \in S_{n}} \theta_{j}\left(\theta_{j} C_{j}^{-1}\right) \\
& \leqslant \sum_{n \geqslant 1} w_{n} \sum_{j \in S_{n}} \theta_{j}=\theta(0) \sum_{n \geqslant 1} w_{n} \\
& <\infty,
\end{aligned}
$$

where we have used Eq. (3.26). The Cauchy-Schwartz inequality shows that

$$
\left(\sum_{j \in S_{n}} \theta_{j}\right)^{2} \leqslant\left(\sum_{j \in S_{n}} C_{j}\right)\left(\sum_{j \in S_{n}} \theta_{j}^{2} C_{j}^{-1}\right) .
$$

The finite energy of $\theta$ implies that $\Sigma_{j \in S_{n}} \theta_{j}^{2} C_{j}^{-1} \rightarrow 0$ as $n \rightarrow \infty$. Since $\theta(0)$ is nonzero, it follows from Eq. (3.26) that

$$
\lim _{n \rightarrow \infty} \sum_{j \in S_{n}} C_{j}=\infty
$$

and hence $C=\infty$ with probability 1 .

Remark 1. Theorem 1 establishes that there is a first-order phase transition from a localized to a delocalized state at the critical points $\sigma_{c}^{*}>0$, where $z \beta\left(\sigma_{c}^{*}\right)=1$. This determines a phase boundary in the space of probability densities that separates the localized and delocalized phases. An interesting question concerns what happens on the phase boundary itself. That is, given a probability density $\rho(v)$ such that $z\left\langle e^{-\sigma^{*} v}\right\rangle=1$, is the steady state localized or delocalized? At present this remains an open problem.

Remark 2. One of the assumptions of theorem 1 is that the drift velocities $v_{i}$ are finite with probability 1 . If this restriction is removed so that there is a nonzero probability that $v_{i}$ is infinite, then one has a bond percolation problem since $f(\infty)=g(\infty)=0$. That is, segments with infinite drift velocities act as broken bonds. It turns out that theorem 1 still holds provided that the statement "with probability 1" in case (ii) is replaced by "with positive probability" [17]. Alternatively, we can state that in case (ii) there is some vertex in $\Gamma$ at which the steady state is delocalized with probability 1. A more detailed discussion of the percolation limit is presented in Sec. IV.

Remark 3. Theorem 1 can be generalized to the case of an irregular unbounded tree $\Gamma$ by defining the branching number of $\Gamma$ according to [17]

$$
\mathcal{B}(\Gamma)=\inf \left\{\zeta>0 ; \inf _{\Pi} \sum_{i \in \Pi} \zeta^{-|i|}=0\right\}
$$

Here $\mathcal{B}(\Gamma)$ is a measure of the average number of branches per vertex of $\Gamma$. For a fixed $\zeta$, one first defines $F(\zeta, \Pi)$ $=\Sigma_{i \in \Pi} \zeta^{-|i|}$, where $|i|$ is the distance from the origin of a given element $i$ of a given cutset $\Pi$. One then finds the smallest value of $\zeta$ for which $F(\zeta, \Pi)$ attains its lower bound of zero for at least one cutset. It is easy to show that $\mathcal{B}(\Gamma)$ $=z$ for a regular tree. Simply take $\Pi$ to be a given generation $n$ such that $F(\zeta, \Pi)=z^{n} \zeta^{-n}$, and use the fact that 
$\lim _{n \rightarrow \infty} z^{n} \zeta^{-n}=0$ if $\zeta<z$. The result then follows since one can also show that no other cutset $\Pi$ gives $F(\zeta, \Pi)=0$ for $\zeta>z$. The branching number is less than or equal to the so-called growth rate

$$
\mathcal{G}(\Gamma)=\lim _{n \rightarrow \infty} \inf M_{n}^{1 / n}
$$

where $M_{n}$ is the number of branches in the $n$th generation. A tree is said to be quasispherical if $\mathcal{B}(\Gamma)=\mathcal{G}(\Gamma)$. For a regular tree we again have $\mathcal{G}(\Gamma)=z$ since $M_{n}=z^{n}$. Another interesting example is a genealogical tree generated by a simple Galton-Watson branching process; starting from the root each vertex has $k$ branches with probability $p_{k}\left(\Sigma_{k} p_{k}=1\right)$. The average number of branches per vertex is $m=\Sigma_{k} k p_{k}$. A well-known result is that the branching process becomes extinct (the associated genealogical tree is finite) with probability 1 if $m \leqslant 1$ [20]. It can be shown that for a branching process with $m>1$ and given that the process does not become extinct, the associated (infinite) genealogical tree is quasispherical and has branching number $m$ with probability 1 [17].

Using definitions (3.29) and (3.30), theorem 1 can now be applied to an irregular tree on replacing $z$ by $\mathcal{G}(\Gamma)$ in part (i) and by $\mathcal{B}(\Gamma)$ in part (ii). In the case of nonquasispherical trees, the theorem is not sufficient to determine the precise location of the localization-delocalization transition point. However, we expect the transition point to be given by $q \beta\left(\sigma^{*}\right)=1$ for some $q \in[\mathcal{B}(\Gamma), \mathcal{G}(\Gamma)]$. Also note that for irregular trees it is no longer possible to construct a DysonSchmidt-type integral equation analogous to Eq. (3.20), since the existence of such an equation relied on the recursive structure of a regular tree.

Remark 4. The proof of theorem 1 exploits the fact that the problem of localization or delocalization of the steady state of the drift-diffusion equation on a tree $\Gamma$ can be mapped onto an analogous problem concerning flows on the tree. A similar connection exists between flows and random walks on $\Gamma[17,18]$. To see this, introduce the transition probabilities $P_{i j}=\operatorname{Prob}[i \rightarrow j]$ between nearest-neighbor vertices on the tree. Denote the first vertex on the shortest path from $i$ to the origin by $i^{\prime}$, and set (for $|i| \geqslant 2$ )

$$
X_{i}=-\ln \frac{P_{i^{\prime} i}}{P_{i^{\prime} i^{\prime \prime}}},
$$

and $X_{i}=-\ln P_{0 i}$ for all $i \in S_{1}$. Assume that $X_{i}$ are independently and identically distributed. Introduce the conductance

$$
C_{i}=\prod_{j \leqslant i} e^{-X_{j}}
$$

Note that the transition probabilities can be recovered from Eq. (3.32) according to

$$
P_{i i^{\prime}}=\frac{C_{i}}{C_{i}+\sum_{j \in \mathcal{I}_{i}} C_{j}}, \quad P_{i^{\prime} i}=\frac{C_{i}}{C_{i^{\prime}}+\sum_{j \in \mathcal{I}_{i^{\prime}}} C_{j}} .
$$

Consider a random walk on $\Gamma$ starting from the origin. The random walk is said to be recurrent if the probability of returning to the origin is equal to 1 . Otherwise the random walk is said to be transient. Let $f_{0}(n)$ be the probability of first returning to the origin in $n$ steps and define the mean recurrence time according to $\mu_{0}=\sum_{n=1}^{\infty} n f_{0}(n)$. A recurrent random walk is said to be null if $\mu_{0}=\infty$ and positive if $\mu_{0}$ $<\infty$. One then has the following result due to Lyons and Pemantle $[17,18]$.

Theorem 2: Consider a random walk on a tree $\Gamma$ with quenched random transition probabilities as described above. Assume that $X_{i}$ is finite w.p.1. Let $\beta(\sigma)=\left\langle e^{-\sigma X}\right\rangle$ and $\beta\left(\sigma^{*}\right)=\min _{0 \leqslant \sigma \leqslant 1} \beta(\sigma)$. (i) If $\mathcal{G}(\Gamma) \beta\left(\sigma^{*}\right)<1$, then the random walk is positive recurrent with probability 1 . (ii) If $\mathcal{B}(\Gamma) \beta\left(\sigma^{*}\right)>1$, then the random walk is transient with probability 1 .

\section{Examples}

We shall now consider the localization-delocalization phase boundary for some particular choices of the velocity probability density $\rho(v)$. In the case of the Bernoulli, Gaussian, and $\Gamma$ distributions, we determine the phase boundary as a curve in the $(\mu, \Delta)$ plane, where $\mu$ and $\Delta$ are the mean and standard deviations, respectively. This is achieved first by finding $\sigma^{*}$ as defined in Eq. (3.23) and then by solving the equation $\beta\left(\sigma^{*}\right)=1 / z$. All three boundary curves meet at the critical point $(\mu, \Delta)=(\ln z, 0)$ since this corresponds to the case of uniform drift; see Eq. (2.12). We then consider an example of a probability density that does not possess any finite moments. Nevertheless, one can still identify parameters characterizing the location and width of the (unimodal) distribution, which play an analogous role to the mean and variance.

Example 1-Bernoulli distribution. Consider a Bernoulli distribution $B\left(p, u_{+}, u_{-}\right)$with

$$
\rho(v)=p \delta\left(v+u_{-}\right)+q \delta\left(v-u_{+}\right), \quad u_{-}, u_{+} \geqslant 0, \quad q=1-p .
$$

From Eq. (3.22),

$$
\beta(\sigma)=p e^{\sigma u_{-}}+q e^{-\sigma u_{+}} .
$$

For this example there are three possibilities.

(i) If

$$
\frac{q u_{+}}{p u_{-}}<1,
$$

then $\sigma^{*}=0, \beta\left(\sigma^{*}\right)=1>1 / z$, and the system is delocalized according to theorem 1(ii).

(ii) If

$$
e^{u_{+}+u_{-}}<\frac{q u_{+}}{p u_{-}}
$$

then $\sigma^{*}=1$ and the phase boundary in $\left(p, u_{+}, u_{-}\right)$-space is given implicitly by the equation

$$
p e^{u_{-}}+q e^{-u_{+}}=z^{-1} .
$$

(iii) If

$$
1<\frac{q u_{+}}{p u_{-}}<e^{u_{+}+u_{-}},
$$




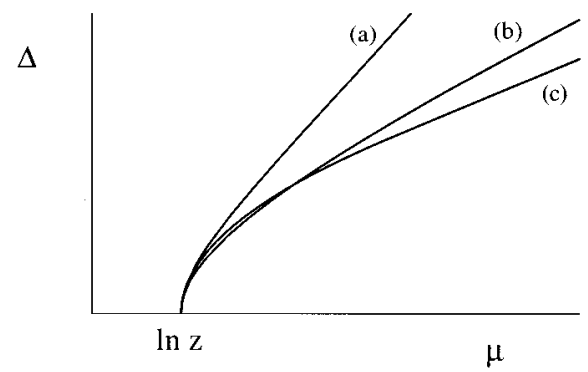

FIG. 3. Phase diagram showing localization-delocalization phase boundaries for various distributions of drift velocities: (a) Bernoulli, (b) $\Gamma$, (c) Gaussian. In each case the system is delocalized in the region on the left of the phase boundary and localized in the right-hand region. The detailed shape of the curves depends on the value of $z$; here we have set $z=2$.

then $0<\sigma^{*}<1$ and the phase boundary is given implicitly by the equation

$$
\frac{q\left(u_{+}+u_{-}\right)}{u_{-}}\left[\frac{p u_{-}}{q u_{+}}\right]^{u_{+} /\left(u_{+}+u_{-}\right)}=z^{-1} .
$$

The phase boundary in the special case $u_{-}=0$, $u_{+}=\bar{v}>0$ is characterized completely by Eq. (3.38), which reduces to a curve in the $(p, \bar{v})$ plane:

$$
\bar{v}(p)=\ln \left(\frac{1-p}{z^{-1}-p}\right) \text {. }
$$

It is useful to consider the corresponding curve in the $(\mu, \Delta)$ plane, where $\mu=q \bar{v}$ is the mean and $\Delta=\sqrt{p q} \bar{v}$ is the standard deviation:

$$
\Delta(\mu)=\sqrt{x(\mu)-\mu^{2}}
$$

where $x(\mu)$ is the solution to the transcendental equation

$$
\left(1-z^{-1}\right) x+\mu^{2} e^{-x / \mu}=\mu^{2}
$$

The trivial solution of Eq. (3.43), $x(\mu)=0$, is excluded since this would give a nonreal variance. There exists a unique nonzero solution to Eq. (3.43) with $x(\mu) \geqslant \mu^{2}$ and $\Delta(\mu)$ real if and only if $\mu \geqslant \ln z$. For $\mu \approx \ln z$ the solution $\Delta(\mu)$ has the approximate form

$$
\Delta(\mu) \approx \ln z\left(\frac{\mu-\ln z}{z-\ln z-1}\right)^{1 / 2} .
$$

The phase boundary in the $(\mu, \Delta)$ plane is shown in Fig. 3(a).

Example 2-Gaussian distribution. Consider the Gaussian distribution $N(\mu, \Delta)$ with density (3.14). The function $\beta(\sigma)$ is given by Eq. (3.15). Here one finds that $\sigma^{*}=\mu / \Delta^{2}$ if $\mu$ $<\Delta^{2}$ and $\sigma^{*}=1$ if $\mu \geqslant \Delta^{2}$. This leads to the following explicit expressions for the phase boundary:

$$
\begin{gathered}
\Delta(\mu)=\sqrt{2(\mu-\ln z)} \text { for } \mu \in[\ln z, 2 \ln z], \\
\Delta(\mu)=\mu / \sqrt{2 \ln z} \text { for } \mu>2 \ln z .
\end{gathered}
$$

The resulting phase boundary is shown in Fig. 3(c).

Example 3- $\Gamma$ distribution. Consider the Gamma distribu- tion $\Gamma(\lambda, b)$ with density

$$
\rho(v)=\frac{\lambda^{b}}{\Gamma(b)} v^{b-1} e^{-\lambda v}, \quad v \geqslant 0,
$$

where $\Gamma(b)$ is the Gamma function

$$
\Gamma(b)=\int_{0}^{\infty} x^{b-1} e^{-x} d x
$$

If $b=1$, then $v$ is exponentially distributed with parameter $v$. Also note that if $\lambda=\frac{1}{2}$ and $b=n / 2$ for some integer $n$, then $v$ is said to have the chi-squared distribution $\chi^{2}(n)$ with $n$ degrees of freedom. Since $v \geqslant 0$ we know that $\sigma^{*}=1$ $[\beta(\sigma)$ is a monotonically decreasing function of $\sigma]$. Thus the phase boundary in the $(\lambda, b)$ plane is given implicitly by

$$
\frac{\lambda^{b}}{(1+\lambda)^{b}}=z^{-1}
$$

Using the fact that the mean $\mu=b / \lambda$ and the variance $\Delta^{2}$ $=b / \lambda^{2}$, Eq. (3.49) can be rewritten as

$$
1+\frac{\Delta^{2}}{\mu}=z^{\Delta^{2} / \mu^{2}}
$$

As in the previous examples, Eq. (3.50) only has a nontrivial solution $\Delta(\mu)$ if $\mu \geqslant \ln z$. In the limit $\Delta \rightarrow 0, \mu \rightarrow \ln z$ and Eq. (3.50) reduces to

$$
\Delta(\mu) \approx \sqrt{2(\mu-\ln z)} \text { for } \mu \approx \ln z .
$$

The phase boundary is shown in Fig. 3(b).

The similarity in the behavior of the $\Gamma$ distribution to the Gaussian distribution close to the critical point $(\ln z, 0)$ can be understood in terms of a cumulant expansion of $\beta(\sigma)$ under the assumption that all the moments of the density $\rho(v)$ are finite. That is, write

$$
\beta(\sigma)=e^{-w(\sigma)},
$$

with

$$
w(\sigma)=\sum_{k=0} \frac{w_{k}}{k !} \sigma^{k},
$$

such that

$$
\begin{gathered}
w_{0}=0, \quad w_{1}=\mu, \quad w_{2}=-\Delta^{2}, \\
w_{3}=\left\langle v^{3}\right\rangle-3 \mu \Delta^{2}-\mu^{3}, \ldots .
\end{gathered}
$$

Assume that $\mu>0$ and the fluctuations are small such that $\mu \gg \Delta^{2}$. Further, assume that $\Delta^{2} \gg w_{k}$ for all $k>2$, which is true for unimodal distributions such as the $\Gamma$ distribution. (In the case of a Gaussian $w_{k} \equiv 0$ for all $k>2$ ). Neglecting third and higher moments in the cumulant expansion (3.53) then leads to the approximation

$$
\beta(\sigma) \approx e^{-\sigma \mu+\sigma^{2} \Delta^{2} / 2} .
$$

Since $\mu \gg \Delta^{2}$, it follows that $\sigma^{*}=1$ and the equation for the phase boundary, $\beta\left(\sigma^{*}\right)=1 / z$, reduces to Eq. (3.51).

Example 4. As our final example we consider a probability density whose moments are all infinite: 


$$
\rho(v)=\frac{a}{2 \sqrt{\pi(v+b)^{3}}} e^{-a^{2} / 4(v+b)}, \quad v>-b, \quad b>0 .
$$

From Eq. (3.22),

$$
\beta(\sigma)=e^{b \sigma-a \sqrt{\sigma}} .
$$

If $a>2 b$, then $\sigma^{*}=1$, and the phase boundary in the $(a, b)$ plane is given by

$$
b(a)=a-\ln z .
$$

On the other hand, if $a<2 b$, then $\sigma^{*}=a^{2} / 4 b^{2}$, and the phase boundary is given by

$$
b(a)=a^{2} / 4 \ln z .
$$

Comparing Eqs. (3.45) and (3.46) with Eqs. (3.58) and (3.59) then shows that the density (3.56) has a phase boundary curve identical to that of a Gaussian $N(a, \sqrt{2 b})$.

It is interesting to consider from the above examples how one can recover the critical phase boundary in one dimension $(z=1)$. The relevant criterion is $\langle v\rangle=0$ (see Sec. III A), which corresponds to the vertical line $\mu=0$. The correct procedure is to define $z=1+\epsilon$, and analytically continue such that $\epsilon \rightarrow 0$. To show that the phase boundaries derived for the examples above tend toward the line $\mu=0$ as $\epsilon \rightarrow 0$, consider a fixed value of $\mu=\delta>\epsilon$, and ask what happens to the standard deviation $\Delta$ as $\epsilon \rightarrow 0$. It is easy to show that $\Delta$ diverges as $1 / \sqrt{\epsilon}$. Since this holds for any $\delta$, it is clear that all the boundary curves collapse to the line $\mu=0$. It also follows that the region of validity of the expansions around the point $\mu=\ln z$ becomes progressively smaller as $\epsilon \rightarrow 0$. The mathematical details concerning this particular issue will be discussed more fully elsewhere [21].

\section{PERCOLATION ON A TREE}

The results presented above have a number of important implications for percolation theory. Although, in essence, already covered in the detailed mathematical work of Lyons [17], it is nevertheless instructive to point out these connections and to discuss their physical interpretation.

The classic problem of bond percolation on an infinite, regular Cayley tree with branching number $z$ is well understood. Branches are randomly occupied (unit capacity) with probability $p$ or left unoccupied (zero capacity) with probability $1-p$. One is interested in establishing whether or not a connection from the origin to infinity is made, i.e., whether or not the origin is linked to a connected cluster of infinite capacity. Arguments based on generating functions (see, e.g., Ref. [1]) show that the critical concentration $p_{c}$ for the formation of an infinite cluster is given by $p_{c}=1 / z$. For $p$ $<p_{c}$ an infinite cluster is never formed; for $p>p_{c}$ there is a nonzero probability that the origin will belong to an infinite cluster, and this probability becomes unity when $p=1$. Thus if the probability of the origin belonging to an infinite cluster is viewed as an order parameter, then geometric bond percolation exhibits a second-order phase transition.

In the present paper we also considered infinite, regular trees, whereby each branch $i$ is characterized by a velocity parameter $v_{i}$ and has an associated capacity (or conductance) $f\left(v_{i}\right) \Pi_{j} g\left(v_{j}\right)$ [Eq. (3.24)]. The localization criterion of theorem $1, z\left\langle e^{-\sigma^{*} v}\right\rangle<1$ (localized) or $z\left\langle e^{-\sigma^{*} v}\right\rangle>1$ (delocalized), is equivalent to whether the total capacity (or conductance) on the tree is finite or infinite (related to normalizability). To make the connection with geometric bond percolation, consider a Bernoulli distribution with density

$$
\rho(v)=p \delta(v)+(1-p) \delta(v-\bar{v}) .
$$

Any branch with $v=0$ that is connected to the origin by other branches with $v=0$ will act as a bond of unit capacity [since $f(0)=g(0)=1$ ]. On the other hand, in the limit $\bar{v} \rightarrow \infty$, each branch with $v=\bar{v}$ will act as a broken bond of zero capacity [since $f(\infty)=0$ ], and will set the capacity of all subsequent branches equal to zero [since $g(\infty)=0$ ]. Thus, in terms of whether the origin belongs to a cluster of finite or infinite capacity, we have an identical model to that of geometric bond percolation. Since the allowed velocities in Eq. (4.1) are non-negative, $\sigma^{*}=1$ and the threshold for the transition is determined by $z\left\langle e^{-v}\right\rangle=1$ (see remark 2 in Sec. II B). It follows immediately that $p_{c} z=1$ or $p_{c}=1 / z$. When $p_{c}<1 / z$ the system is localized (has finite capacity) with probability 1 , and $F^{*}=0$ [see Eq. (3.10)]. When $p_{c}>1 / z$ the system may either be localized (finite capacity) or delocalized (infinite capacity) and $0<F^{*}<1$ (see again remark 2 in Sec. III B), which corresponds to the transition being second order with $F^{*}$ identified as an order parameter. From the integral equation (3.21) we obtain $M(s)=p M(s)^{z} e^{-s}+(1$ $-p)$ [noting that $M(0) \equiv 1$ ], from which one can derive the value of the order parameter $F^{*}=1-\lim _{s \rightarrow 0} M(s)$. For instance, when $z=2$ one obtains the well-known result that $F^{*}=0$ for $p<\frac{1}{2}$ and $F^{*}=(2 p-1) / 2 p$ for $p>\frac{1}{2}$ [1].

The above derivation puts the nature of the phase transition of geometric bond percolation into context. For almost all parametrized velocity distributions (i.e., those which do not allow $v$ to be infinite with any finite probability), the transition governing whether the total capacity is finite or infinite is strictly first order, the order parameter $F^{*}$ jumping from zero to unity as some parameter is varied. The secondorder nature of the geometric bond percolation problem is thus a unique feature of the fact that allowing infinite velocities effectively destroys the connectivity of the underlying tree. One can, in fact, cast the geometric problem in such a way that it too exhibits a first-order rather than second-order transition, by noting that the probability that an infinite cluster exists somewhere on the tree for $p>p_{c}$ is 1 (although the origin may not, of course, belong to it if the tree is suitably disconnected).

We now have the basis for analyzing more general models of percolation on tree structures. Consider once again the above Bernoulli distribution, but this time choose $\bar{v}$ to be finite. Define $\epsilon=e^{-\bar{v}}$. The transition criterion $z\left\langle e^{-\sigma^{*} v}\right\rangle=1$ yields a critical transition probability of

$$
p_{c}=\frac{1-z \epsilon}{z(1-\epsilon)} .
$$

The physical interpretation of this two-component model of percolation is as follows. One has an infinite, regular tree 
along the branches of which some flow process is taking place. Some branches are relatively open (those with $v=0$ ); others are very constrictive (those with $v=\vec{v}$ ). The constrictive links affect other links downstream from the origin; this is taken into account through the multiplicative nature of the model. If $p<p_{c}$ then flow between the origin and infinity is impossible. On the other hand, if $p>p_{c}$, then finite flow will occur with probability 1 . Note the emphasis, "will occur" rather than "might occur," since the transition is now strictly first order rather than second order. This interpretation in terms of flows is quite natural, given the derivation of some of our results in terms of flow theory on networks and an obvious natural connection to random resistive or capacitive networks. From the above model we note that as $\epsilon \rightarrow 0 \quad(\bar{v} \rightarrow \infty)$, so $p_{c} \rightarrow 1 / z \quad$ (the geometric limit), while for $\epsilon \geqslant 1 / z,(\bar{v} \leqslant \ln z)$ no transition is possible (the system is always percolating). Many alternative models of percolating processes can be studied by choosing different velocity densities $\rho(v)$, and details of the behavior on the nonpercolating (localized) side of the transition point may be obtained from the integral equation (3.21). Exploring the consequences of this connection with percolation theory deserves further study.

Finally, the theorems derived by Lyons have implications for percolation on trees with random branching numbers at each branching site. First, one creates the underlying tree structure upon which percolation is to take place. Suppose we have a local branching probability $p_{k}$ with $\Sigma_{k} p_{k}=1$. From the theory of genealogical trees associated with Galton-Watson branching processes [20], we know that the tree will always be finite in extent (extinction will occur with probability 1) if $m \equiv \Sigma_{k} k p_{k} \leqslant 1$. Clearly such situations are not particularly interesting as regards percolation. If $m>1$ then the probability for generating an infinite (although irregular) tree is nonzero (the probability of nonextinction is greater than zero), which is reminiscent of the second-order transition discussed above. Let us imagine that we have $m$ $>1$ and that we have generated an infinite, irregular tree. For pure geometric percolation, one now occupies the branches with probability $p$ or leaves them unoccupied with probability $1-p$. The percolation threshold governing whether the origin belongs to an infinite cluster is then given by $p_{c}$ $=1 / \mathrm{m}$ [17]. More generally, the full transition criterion be- comes $m\left\langle e^{-\sigma^{*} v}\right\rangle=1$. Thus in the case of the above twocomponent model defined on a genealogical tree of a GaltonWatson process, we would have

$$
p_{c}=\frac{1-m \epsilon}{m(1-\epsilon)} .
$$

\section{CONCLUSIONS}

In this paper we have examined drift diffusion on a regular tree with quenched, random drift velocities on its branches, and derived criteria for whether the system is localized or delocalized, given an arbitrary distribution for the drift velocities. We have also presented examples of how the system can undergo a phase transition from a localized to a delocalized state as some parameter defining a family of velocity distributions is varied. Such transitions are generically first order rather than second order. A formal link with conventional percolation theory has been made, and we have indicated how some (but not all) of the results can be extended to the case of irregular trees.

Once one has established that, for a given velocity density $\rho(v)$, the system is localized, it is natural to ask questions about the nature of the localized state. For example, what is the distribution of the residual amplitude at the origin, given an initial unit impulse? Or, can one define a suitably averaged localization length? Or, to what extent is self-averaging relevant in the system? We have not attempted to answer these questions in this paper, although the integral equations we have presented provide a natural starting point as regards the first of them. Direct numerical simulation is an obvious approach, but this is not straightforward, especially for systems that are "only just localized" (it is extremely difficult to establish whether a system is localized or delocalized near the phase boundary by numerical methods). This is one reason why having exact mathematical criteria for the transition points is so valuable.

Finally, the results presented relate to the convergence or otherwise of quite general random, multiplicative sequences with an underlying treelike structure. As such, we feel that they are likely to be applicable to many other physical problems besides the one considered here.
[1] Fractals and Disordered Systems, edited by A. Bunde and S. Havlin (Springer-Verlag, Heidelberg, 1991).

[2] J.-P. Bouchaud and A. Georges, Phys. Rep. 195, 127 (1990).

[3] J. M. Ziman, Models of Disorder (Cambridge University Press, Cambridge, 1979).

[4] R. J. Baxter, Exactly Solved Models in Statistical Mechanics (Academic, London, 1982).

[5] P. C. Bressloff, V. M. Dwyer, and M. J. Kearney, Phys. Rev. Lett. 77, 5075 (1996).

[6] D. Cassi, Europhys. Lett. 9, 637 (1989).

[7] P. Sibani, Phys. Rev. B 34, 3553 (1986).
[8] C. Aslangul, M. Barthélémy, N. Pottier, and D. Saint-James, Europhys. Lett. 15, 251 (1991).

[9] P. C. Bressloff, V. M. Dwyer, and M. J. Kearney, J. Phys. A 29, 6161 (1996).

[10] Ya G. Sinai, Lect. Notes Phys. 153, 12 (1982).

[11] B. Derrida and Y. Pomeau, Phys. Rev. Lett. 48, 627 (1982).

[12] C. de Calan, J. M. Luck, Th. M. Nieuwenhuizen, and D. Petritis, J. Phys. A 18, 501 (1985).

[13] B. Derrida and H. J. Hilhorst, J. Phys. A 16, 2641 (1983).

[14] F. Solomon, Ann. Prob. 3, 1 (1975).

[15] H. Kesten, Acta Math. 131, 208 (1973). 
[16] H. Kesten, M. V. Kozlov, and F. Spitzer, Comp. Math. 30, 169 (1975).

[17] R. Lyons, Ann. Prob. 18, 931 (1990).

[18] R. Lyons and R. Pemantle, Ann. Prob. 20, 125 (1992).

[19] L. R. Ford and D. R. Fulkerson, Flows in Networks (Princeton
University Press, Princeton, NJ, 1962).

[20] G. R. Grimmett and D. R. Stirzaker, Probability and Random Processes (Clarendon, Oxford, 1988).

[21] M. J. Kearney, V. M. Dwyer, and P. C. Bressloff, J. Phys. A (to be published). 